\title{
Skaleneffekte bei Verwaltungs- und Verfahrenskosten der Unfallversicherungsträger der öffentlichen Hand
}

\author{
Einsparpotenzial; Gesetzliche Unfallversicherung in Deutschland; Panelanalyse; Reform der öf-
} fentlichen Unfallkassen; Skaleneffekte; Verwaltungs- und Verfahrenskosten

Die gesetzliche Unfallversicherung wurde im Rahmen des Unfallversicherungsmodernisierungsgesetzes (UVMG) zum 1.1.2009 grundlegend reformiert. Ein wesentliches Ziel der Reformen war es, durch Fusionen der Versicherungsträger die Verwaltungs- und Verfahrenskosten je Versicherten zu reduzieren, also Skaleneffekte zu realisieren. Allerdings gab es zum Zeitpunkt der Reform keinerlei empirische Evidenz, dass bedeutsame Skaleneffekte zu erwarten wären. Basierend auf Daten der Jahre 1998-2007 wird die Frage untersucht, ob große Unfallkassen geringere Verwaltungs- und Verfahrenskosten je Versicherten aufwenden müssen als kleine. Mittels Paneldatenanalyse kann über mehrere Spezifikationen hinweg robust gezeigt werden, dass die Höhe der Verwaltungs- und Verfahrenskosten je Versicherten negativ von der Größe des Trägers (gemessen durch die Anzahl der Versicherten) abhängt. Im statistischen Mittel sinken die Verwaltungs- und Verfahrenskosten je Versicherten um ca. 33 Cent, wenn die Anzahl der Versicherten um 100.000 zunimmt. Bei mittleren Kosten von ca. 6,54 $€$ je Versicherten über den betrachteten Zeitraum und durchschnittlich 960.000 Versicherten je Unfallkasse ist dieses Einsparpotential erheblich. Bei einer Steigerung der Kassengröße um ca. 600.000 Versicherte (um eine Standardabweichung) besteht damit ein Einsparpotenzial von ca. $30 \%$.

\section{Einführung}

Die gesetzliche Unfallversicherung war in den letzten Jahren verstärkt Gegenstand politischer Interventionen und Reformen. Trotz der politischen Diskussion um diesen bedeutenden Zweig der Sozialversicherungen wird die gesetzliche Unfallversicherung in der Öffentlichkeit noch immer kaum wahrgenommen. Sie gliedert sich in die zwei Teilbereiche der gewerblichen Berufsgenossenschaften und der öffentlichen Unfallkassen. Erstere decken den erwerbswirtschaftlichen Bereich ab und versicherten im Jahr 2009 ca. 47 Millionen Personen. Letztere setzen sich aus der Allgemeinen Unfallversicherung (AUV: 10,9 Millionen Versicherte) und der Schülerversicherung (SUV: 17 Millionen Versicherte) zusammen und waren 2009 für insgesamt 27,9 Millionen Versicherte verantwortlich. ${ }^{2}$ Diese Analyse fokussiert sich ausschließlich auf die Unfallkassen als Unfallversicherungsträger der öffentlichen Hand. Aufgrund der hohen Zahl der Versicherten

\footnotetext{
1 Prof. Dr. Ashok Kaul ist Inhaber des Lehrstuhls für Wirtschaftspolitik an der Universität des Saarlandes. Dr. Gregor Pfeifer, Dipl.-Kfm. Manuel Schieler und Dr. Stefan Witte sind dort wissenschaftliche Mitarbeiter. Korrespondierender Autor ist Ashok Kaul (ashok.kaul@mx.uni-saarland.de). Postadresse: Prof. Dr. Ashok Kaul, Lehrstuhl für Wirtschaftspolitik, Universität des Saarlandes, Campus Gebäude C3.1, 66123 Saarbrücken.

2 Für weitere Informationen zu den Unfallversicherungsträgern der öffentlichen Hand vgl. Kaul/Haag (2008).
} 
und der Tatsache, dass die Kassen durch den Steuerzahler finanziert werden, scheint eine effiziente strukturelle Gestaltung des Trägers unabdingbar. Aus diesem Grund sind nun seit einigen Jahren Forderungen zur grundlegenden Umgestaltung des deutschen Systems der Unfallversicherung an die Politik ergangen und von dieser aufgegriffen worden (Bund-Länder-Arbeitsgruppe 2006).

Die Thematik wurde darüber hinaus in Beratung und Forschung diskutiert, wobei insbesondere Bürokratieabbau, Senkung der Verwaltungskosten und Verringerung der Trägerzahl als Quintessenz der Analysen empfohlen wurden. ${ }^{3}$ Hierbei war insbesondere die Reduktion der Trägerzahl auf einen zentralen Träger für die öffentliche und gewerbliche Unfallversicherung ein wesentlicher Optimierungsvorschlag (Rürup/Steinmeyer 2006). Zwar wurde dieser Forderung noch nicht konkret Rechnung getragen, die Entwicklungen der letzten zwei Jahrzehnte zeigen jedoch klare Konzentrationsbemühungen. Die Zahl von ursprünglich 76 Unfallkassen im Jahr 1990 hat sich über die Zeit aufgrund von Fusionen reduziert, wobei vorrangig regionale Zusammenschlüsse erfolgten. Nach dem Unfallversicherungsmodernisierungsgesetz (2009) ist die Anzahl zum heutigen Tag auf 17 Unfallkassen festgelegt. ${ }^{4}$

Der Weg hin zu mehr Konzentration bzw. einer Einheitsunfallversicherung wurde durch die Ergebnisse einer Arbeit von Kaul und Haag (2008) zum Teil in Frage gestellt: Anhand einer Querschnittsdatenanalyse, basierend auf den Geschäfts- und Rechnungsergebnissen der Unfallversicherungsträger der öffentlichen Hand aus dem Jahr 2005, konnten die Autoren keine Hinweise für effizienteres Arbeiten größerer Träger finden. Jenes Ergebnis könnte allerdings auf eine zu kleine Datenbasis und Verzerrungen bei der Schätzung zurückzuführen sein. Ziel ist nun, die Frage nach der Existenz von Skaleneffekten anhand einer größeren Zeitspanne und adäquater Methodik erneut zu prüfen. Können bei der hier durchgeführten Analyse Skaleneffekte nachgewiesen werden, so kann dies nicht nur als nachträgliche Rechtfertigung der bereits erfolgten Zusammenschlüsse gesehen werden, sondern auch Indizien für eine Quantifizierung zukünftiger Einsparpotenziale liefern.

Die Arbeit ist wie folgt strukturiert: In Kapitel 2 wird die Datenbasis beschrieben, Abschnitt 2.1 geht auf die zentralen Variablen und ihre Benutzung ein, während Abschnitt 2.2 grafische Evidenz zu den wesentlichen Zusammenhängen der Variablen liefert. In Kapitel 3 werden unterschiedliche (Panel-)Regressionsmethoden diskutiert, bevor unser Modell in Kapitel 4 spezifiziert wird. In Kapitel 5 werden die wesentlichen Ergebnisse sowie die Tragweite der geschätzten Werte dargelegt und diskutiert. Teil 6 liefert ein Fazit und einen kurzen Ausblick.

3 Vgl. Roland Berger Strategy Consultants (2005) und Sopoaktuell (2006).

4 Die Frage nach einer erfolgreichen Implementierung des UVMG kann im Rahmen dieser Ausführung nicht beantwortet werden, da die Datenbasis den hierfür relevanten Zeitraum nicht umfasst und eine wissenschaftlich akkurate Evaluation des Gesetzes aufgrund der zahlreichen Umstrukturierungen innerhalb der Kassen nicht ohne Weiteres durchführbar wäre. Vielmehr liefert die vorliegende Studie eine Aussage zum Status Quo vor der Gesetzesverkündung, wodurch die Intention und die Motivation der Gesetzgeber nachträglich bewertet werden können. 


\section{Daten}

Die Datenbasis umfasst die Gesamtheit der öffentlichen Unfallkassen innerhalb des Zeitraumes von 1998-2007 und wurde auf Grundlage deren jeweiliger Geschäfts-, Jahres- und Abschlussberichte erstellt. ${ }^{5}$

Die Einschränkung des Panels auf den Zeitraum ab dem Jahr 1998 ist aus zwei Gründen sinnvoll. Erstens sollen die verwendeten Daten möglichst aktuell sein um die Implikationen der Ergebnisse zeitnah verwenden zu können und weiterhin relevante Aussagen zu treffen. Zweitens darf die Datenbasis, um Skaleneffekte ermitteln zu können, nicht durch die im Zeitverlauf angefallenen Fusionsvorgänge verzerrt werden. Da zum 1.1.1998 eine Welle an Fusionen ${ }^{6}$ stattfand, bietet sich ein Schnitt an dieser Stelle an.

Neben der zeitlichen Einschränkung wird auch die Anzahl bzw. Auswahl der Kassen innerhalb der Analyse eingegrenzt. Dies ist einer gewollten Homogenität zwischen den Unfallkassen geschuldet. Die Bundesunmittelbaren Unfallkassen (Unfallkasse des Bundes, Eisenbahnunfallkasse und Unfallkasse Post und Telekom) sowie die Feuerwehrunfallkassen werden daher nicht in die Analyse mit einbezogen. Ihre Versicherten- und Risikostrukturen würden mögliche Erkenntnisse verfälscht wiedergeben.

Ebenfalls nicht berücksichtigt werden die Unfallkasse Hamburg sowie zwei Datenpunkte der Bayerischen GUV. Hier liegen zu starke Schwankungen der Verwaltungs-und Verfahrenskosten aufgrund gebildeter (außerordentlicher) Rückstellungen vor. Außerdem schließen wir die Unfallkasse Baden-Württemberg aus, da hier eine Fusion innerhalb der betrachteten Jahre vorliegt. $^{7}$

Wir betrachten somit 20 öffentliche Unfallkassen über einen Zeitraum von 1998-2007, wodurch der Datensatz insgesamt 198 Beobachtungen umfasst (vgl. Anhang: Tabelle 2).

\section{Zentrale Variablen und ihre Verwendung}

Als zentraler Treiber für die Verwaltungs- und Verfahrenskosten kann die Zahl der gemeldeten Unfälle vermutet werden. Die Größe gemeldete Unfälle wird jedoch erst ab dem Berichtsjahr 2003 in den Geschäftsergebnissen ausgewiesen. Somit liegen die gemeldeten Unfälle ausschließlich für einen kleinen Abschnitt des Beobachtungszeitraums vor. Durchschnittlich wurden in diesem Zeitraum 11.742 Unfälle innerhalb der AUV gemeldet während die Fallzahlen in der SUV um ein Vielfaches höher, nämlich bei 66.009 Unfällen lagen.

Die beste Proxy-Variable für gemeldete Unfälle ist die Anzahl der Versicherten. ${ }^{8}$ Dieses Maß ist einerseits allgemein am wenigsten anfällig für Messfehler und bildet andererseits die gemeldeten Unfälle mit einer Korrelation von mehr als 0,9 sehr zuverlässig ab. Wir benutzen daher die Va-

\footnotetext{
5 Sämtliche monetären Werte des Datensatzes wurden mithilfe des Konsumentenpreisindex zum Basisjahr 2005 deflationiert.

6 Insgesamt wurden 11 Fusionen zum 1.1.1998 durchgeführt.

7 Der Badische GUVV, der Württembergische GUVV, die Badische Unfallkasse und die Württembergische Unfallkasse fusionierten zum 1.1.2003 zur „Unfallkasse Baden-Württemberg“.

8 aber nicht die Anzahl der meldepflichtigen Unfälle, sondern.
} 
riable Anzahl Versicherte anstelle der gemeldeten Unfälle als wichtigste erklärende Variable für die Verwaltungs- und Verfahrenskosten. Diese liegt für alle Perioden unseres Beobachtungszeitraums vor.

Andere Variablen - z. B. gemeldete und meldepflichtige Unfälle -, die als Kostentreiber in Frage kommen und gegebenenfalls auf Größeneffekte schließen lassen, sind mit der von uns verwendeten Variable hoch korreliert und können daher nicht als zusätzliche Regressoren im Modell berücksichtigt werden.

Weiterhin muss für die Anzahl der versicherten Schüler kontrolliert werden. Dies ist nötig, da Schüler mehr Unfälle verursachen, jedoch zu geringeren Kosten und sich die Unfälle dieser beiden Sparten der öffentlichen Unfallversicherung unterschiedlich auf die Verwaltungs- und Verfahrenskosten auswirken. ${ }^{9}$

Dieser Umstand wird in unserem Modell berücksichtigt, indem wir die Anzahl Versicherte (gesamt) verwenden, zusätzlich allerdings mittels des prozentualen Anteils von Schülern an der Gesamtzahl der Versicherten kontrollieren.

Da Kassen mit höheren Präventionsausgaben auch höhere Verwaltungs- und Verfahrenskosten haben sollten (Kaul/Haag 2008, S. 258 f.) erweitern wir die Regression noch um die Variable Präventionsintensität, welche über den Quotienten aus Präventionsausgaben und Zahl der Versicherten (gesamt) gebildet wird.

\section{Grafische Darstellungen}

Abbildung 1 enthält eine Übersicht über Verwaltungs- und Verfahrenskosten je Versicherten (in Euro) pro Kasse. Die Durchschnittswerte unserer abhängigen Variablen bewegen sich zwischen etwa 4,30 Euro und 9 Euro je Versicherten.

Es wird ersichtlich, dass nicht nur die Unterschiede zwischen den Kassen sehr groß sind - die Unfallkasse Mecklenburg-Vorpommern beispielsweise verursacht im Schnitt mehr als doppelt so hohe Verwaltungs- und Verfahrenskosten wie die Bayerische Landesunfallkasse -, sondern auch eine große Streuung innerhalb der einzelnen Kassen über die Jahre auftritt. Hier sind vor allem die „teureren“ Kassen stärker betroffen.

9 Während die Meldepflicht in der AUV klar definiert ist, müssen in der SUV sämtliche Unfälle gemeldet werden, was den Verwaltungsaufwand des durchschnittlichen Unfalls nach unten zieht. Des Weiteren sind die durchschnittlichen Schülerunfälle leichte Unfälle, die nur geringen Verwaltungsaufwand bedeuten. Vgl. hierzu Kaul/ Haag (2008). 


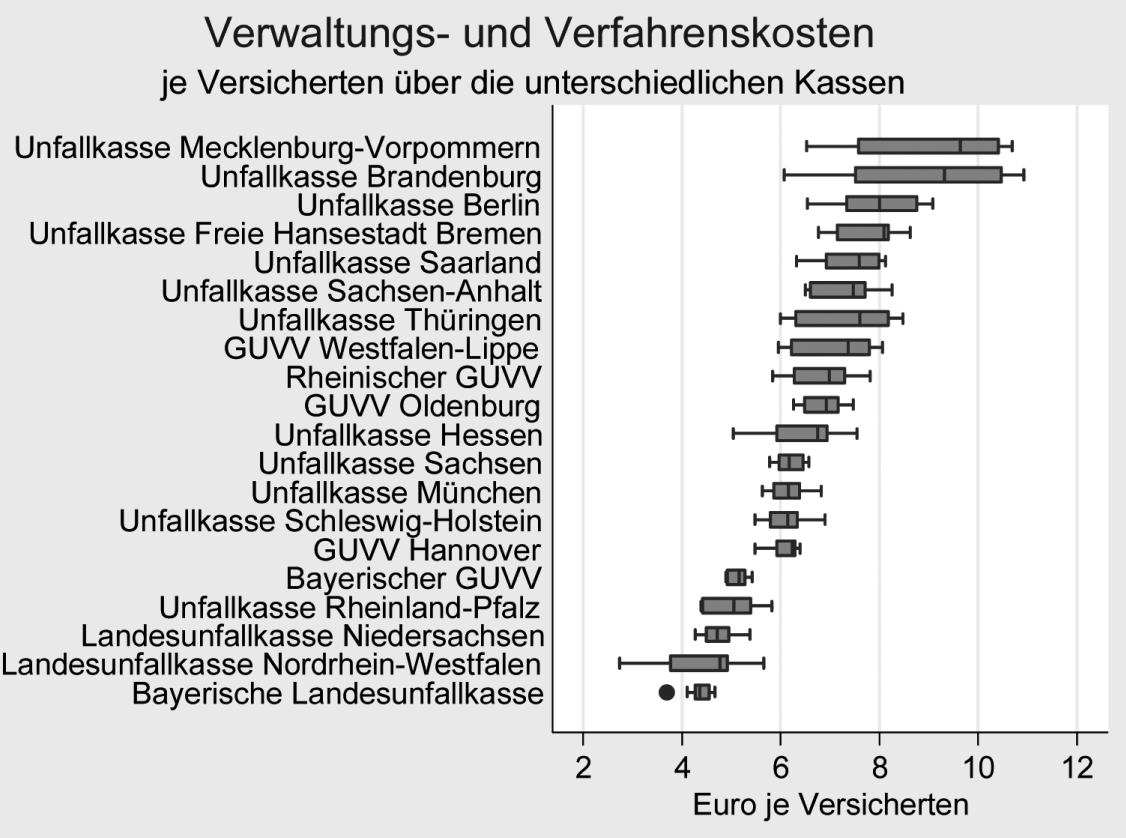

Abbildung 1: Streuung der Verwaltungs- und Verfahrenskosten je Versicherten (in Euro) für alle Kassen über die Jahre 1998-2007

Quelle: Eigene Darstellung. Datenquelle: Geschäfts-, Jahres- und Abschlussberichte der Unfallkassen 1998-2007.

Abbildung 2 zeigt in einem Streudiagramm die Verwaltungs- und Verfahrenskosten je Versicherten (in Euro) gegen die Gesamtzahl der Versicherten sowie die dazugehörige Ausgleichsgerade. Es ist außerdem ersichtlich, dass im Größenbereich zwischen 500.000 und 1.000.000 Versicherten die Durchschnittskosten höher sind und stärker streuen. Insgesamt zeigen sich hier ein negativer Zusammenhang und damit bereits ein erster Hinweis auf Skaleneffekte. 


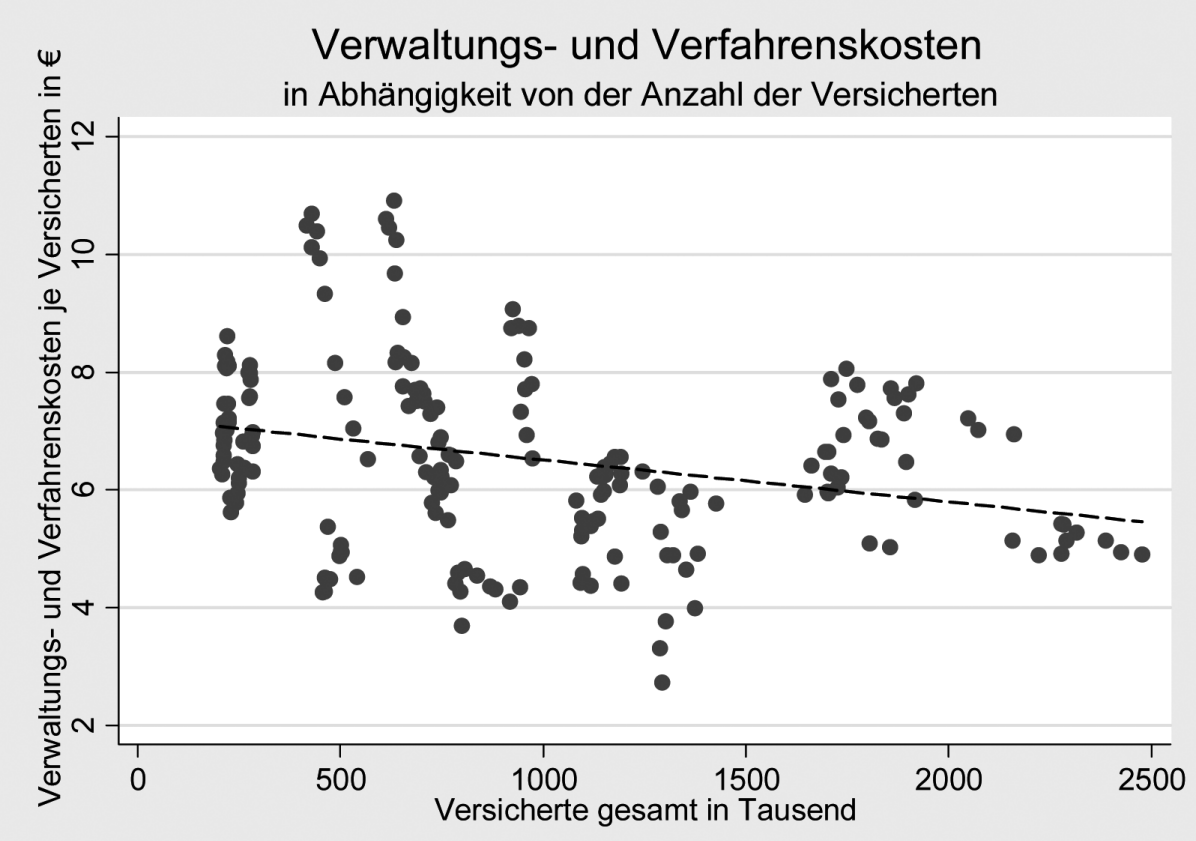

Abbildung 2: Verwaltungs- und Verfahrenskosten der einzelnen Unfallkassen je Versicherten (in Euro) in Abhängigkeit von der Anzahl Versicherte gesamt

Quelle: Eigene Darstellung. Datenquelle: Geschäfts-, Jahres- und Abschlussberichte der Unfallkassen 1998-2007.

Abbildung 3 zeigt den Zusammenhang zwischen Verwaltungs- und Verfahrenskosten je Versicherten (in Euro) und dem prozentualen Anteil der Schüler an der Anzahl der Versicherten insgesamt inklusive Ausgleichsgerade. Im Allgemeinen gilt: je mehr Schüler in einer Unfallkasse versichert sind (relativ zur Anzahl der allgemein Versicherten), desto niedriger schlagen sich die Verwaltungs- und Verfahrenskosten je Versicherten bei der entsprechenden Kasse nieder. Diese Beobachtung liefert Evidenz für den bereits bei der Variablenspezifizierung erwähnten Umstand, dass Schülerunfälle verhältnismäßig geringere Kosten verursachen. 


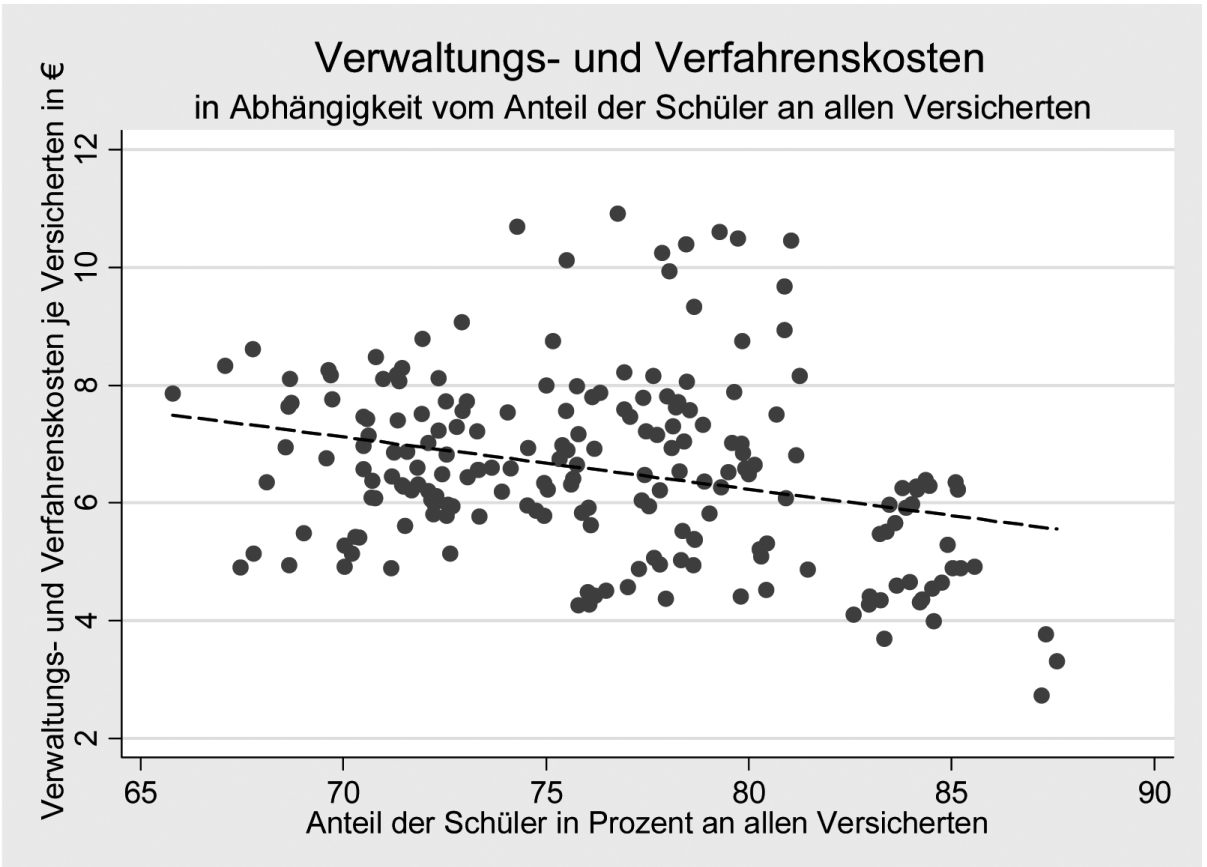

Abbildung 3: Verwaltungs- und Verfahrenskosten je Versicherten (in Euro) in Abhängigkeit vom Anteil der Schüler an der Anzahl Versicherte gesamt

Quelle: Eigene Darstellung. Datenquelle: Geschäfts-, Jahres- und Abschlussberichte der Unfallkassen 1998-2007.

In der Regressionsanalyse (vgl. Kapitel 3) verwenden wir daher (u. a.) beide Variablen als Regressoren: die Anzahl der Versicherten gesamt (AUV und SUV), sowie den Anteil der Schüler an den Versicherten. Wenn Skaleneffekte vorliegen, zeigen diese sich linear in der Gesamtzahl der Versicherten. Würden wir allerdings einzeln auf Versicherte der allgemeinen Unfallversicherung und der Schülerunfallversicherung regressieren, könnten die jeweiligen Größen nicht die Grenzkosten für einen zusätzlich Versicherten abbilden.

Abbildung 4 zeigt ein Streudiagramm mit Ausgleichsgerade, in dem die Verwaltungs- und Verfahrenskosten je Versicherten den Präventionsausgaben je Versicherten (der Präventionsintensität) gegenübergestellt werden. Hier zeigt sich schließlich ein positiver Zusammenhang: je höher die Ausgaben für Prävention, desto größer die von den jeweiligen Unfallkassen zu tragenden Verwaltungs- und Verfahrenskosten. 


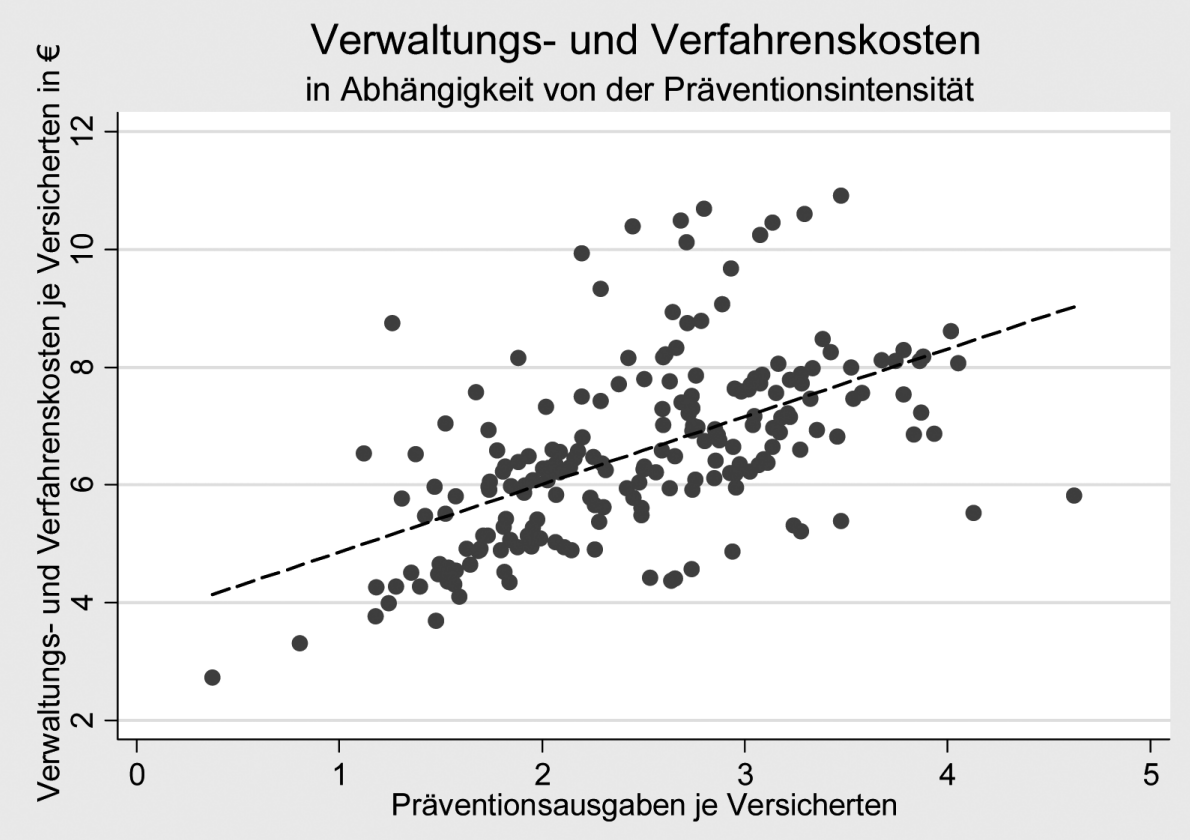

Abbildung 4: Verwaltungs- und Verfahrenskosten je Versicherten in Abhängigkeit von den Präventionsausgaben je Versicherten (jeweils in Euro)

Quelle: Eigene Darstellung. Datenquelle: Geschäfts-, Jahres- und Abschlussberichte der Unfallkassen 1998-2007.

\section{Diskussion alternativer (Panel-)Regressionsmethoden}

Die Struktur der Daten (20 Kassen über den Zeitraum von 10 Jahren) erlaubt grundsätzlich die Anwendung unterschiedlicher Schätzmethoden. Die einfachste Vorgehensweise - und der logische Ausgangspunkt - ist eine klassische gepoolte KQ-Regression. Hierbei würde ein gleiches Absolutglied für alle Beobachtungsträger und Perioden allerdings implizieren, dass kein Fall von Heterogenität über die Träger- und/oder die Zeit vorliegt. Das Modell ignoriert damit die Paneldimension und wäre nur dann anwendbar, wenn die Varianz des Fehlerterms über die Kassen und Jahre als homogen angenommen werden kann (Greene 2008, S. 182). Abbildung 2 liefert klare Hinweise darauf, dass diese Voraussetzung nicht erfüllt ist. Tests auf Heteroskedastie bestätigen diese Annahme. ${ }^{10}$

Folglich ist die Panelstruktur der Daten im Schätzmodell zu berücksichtigen. Entsprechend der Modellspezifikationen mit Verwaltungs- und Verfahrenskosten je Versicherten als abhängige Variable sind statische Panelmethoden wie das Fixed-Effects-Modell oder das Random-Effects-

10 Der ,,residuals-versus-fittet-plot“ weist eindeutig auf Heteroskedastie hin. Sowohl der Breusch-Pagan- als auch der White-Test verwerfen die Nullhypothese der Homoskedastie. 
Modell adäquat. Die Frage welches der beiden Modelle zu wählen ist, wird in der einschlägigen Literatur diskutiert. (Baltagi 2008, S. 21 f.; Greene 2008, S. 183 f.; Kennedy 2009, S. 284 f.; Albers et. al. 2009, S. 263ff.).

Das Fixed-Effects-Modell betrachtet im Gegensatz zur gepoolten Regression ein separates Absolutglied für jeden Beobachtungsträger (Greene 2008, S. 183). Die Fixed-Effects-Schätzung ist auch dann konsistent und verzerrungsfrei, wenn unbeobachtbare Heterogenität vorliegt, ${ }^{11}$ da diese zeitinvarianten Größen durch die Fixed-Effects-Schätzung (vorsätzlich) eliminiert werden. Solche Effekte werden absichtlich aus dem Modell entfernt, da sie unbeobachtet sind und daher als Teil des Fehlerterms die Schätzung verzerren würden - so bspw. im Falle einer gepoolten KQSchätzung. ${ }^{12}$

Der große Vorteil der Fixed-Effects-Schätzung liegt also in der Kontrolle für unbeobachtete Heterogenität bzw. der damit einhergehenden Endogenität. Soll die Regression noch den zeitspezifischen Effekt berücksichtigen, kann eine dementsprechende Konstante hinzugefügt werden (Greene 2008, S. 197).

Für Zufallsstichproben aus einer großen Grundgesamtheit mit dem Ziel, Inferenz für diese Grundgesamtheit zu betreiben, eignet sich hingegen ein Random-Effects-Modell (Baltagi 2008, S. 17). Hierbei muss allerdings zwingend angenommen werden, dass die latenten Effekte mit keinem der Regressoren korreliert sind, da diese bei der Random-Effects-Schätzung gerade nicht eliminiert werden. Nur wenn diese Annahme gilt, ist der Random-Effects-Schätzer konsistent und nicht verzerrt. $^{13}$

Diese formale Voraussetzung, wonach die unbeobachteten, zeitinvarianten Größen mit allen Regressoren unkorreliert sein müssen, wurde in Form eines Hausman-Tests überprüft. Dieser lehnt, auch bei robuster Ausführung, ${ }^{14}$ stets die Verwendung eines Random-Effects-Modells ab. Demzufolge wird für die hier durchzuführende Datenanalyse eine Fixed-Effects-Schätzung verwendet, welche die Unterschiede zwischen den einzelnen Unfallkassen (über die Zeit) systematisch einbezieht.

\section{Spezifikationen des Fixed-Effects-Modells}

Analog der oben diskutierten Eigenschaften werden folgende Spezifikationen auf unser FixedEffects-Modell angewandt:

In der ersten Spezifikation testen wir auf Skaleneffekte, indem wir die Verwaltungs- und Verfahrenskosten je Versicherten $(k v v)$ auf die gesamte Versichertenzahl ${ }^{15}(V G E S)$ regressieren.

11 Sog. latente Effekte, welche mit den Regressoren korreliert sind. Die unbeobachtete Heterogenität führt somit auch stets zu einem Endogenitätsproblem.

12 Die gepoolte KQ-Schätzung ist hier allerdings auch wegen des bereits angesprochenen Problems der Heteroskedastie nicht zu verwenden.

13 Der Fixed-Effects-Schätzer dagegen ist stets konsistent; sowohl wenn die latenten Effekte mit den Regressoren korreliert sind als auch wenn keine Korrelation vorliegt. Im letzten Fall allerdings ist der Random-EffectsSchätzer effizienter.

14 Die robuste Version des Hausman-Tests kontrolliert für Heteroskedastie.

15 Es ist zu beachten, dass in allen Spezifikationen die Variable Versichertenanzahl Gesamt in 1000 eingeht. Dies dient vor allem der optischen Vereinfachung der Regressionsergebnisse, da die berechneten Koeffizienten oftmals im Cent-Bereich liegen. 
Hierbei gehen die Werte pro Kasse $(i)$ und Jahr $(t)$ in die Regression ein. Für jede Kasse $(i)$ wird ein eigenes Absolutglied $\alpha$ geschätzt. $\epsilon_{\mathrm{it}}$ stellt den Fehlerterm dar.

$k v v_{\mathrm{it}}=\alpha_{\mathrm{i}}+\beta_{1} \cdot V G E S_{\mathrm{it}}+\epsilon_{\mathrm{it}}$

Daraufhin erweitern wir das Modell um den prozentualen Anteil der Schüler an allen Versicherten (ant_s). Wir kontrollieren damit direkt dafür, dass verhältnismäßig mehr Schüler in einer Unfallkasse weniger zusätzliche Kosten verursachen:

$k v v_{\mathrm{it}}=\alpha_{\mathrm{i}}+\beta_{1} \cdot V G E S_{\mathrm{it}}+\beta_{2} \cdot a n t_{-} s+\epsilon_{\mathrm{it}}$

In der nächsten Spezifikation wird zusätzlich die Kosten für Präventionsausgaben je Versicherten (kpr) berücksichtigt:, ${ }^{1617}$

$k v v_{\mathrm{it}}=\alpha_{\mathrm{i}}+\beta_{1} \cdot V G E S_{\mathrm{it}}+\beta_{2} \cdot a n t_{-} s+\beta_{3} \cdot k p r_{\mathrm{it}}+\epsilon_{\mathrm{it}}$

In der vierten Spezifikation berücksichtigen wir generell mögliche Unterschiede über den Zeitverlauf bzw. Makrotrends, indem wir für alle Jahre des Beobachtungszeitraums mit Jahresdummies (y) kontrollieren. ${ }^{18}$ Das Jahr 1998 fungiert hierbei als Basisjahr.

$k v v_{\mathrm{it}}=\alpha_{\mathrm{i}}+\beta_{1} \cdot V G E S_{\mathrm{it}}+\beta_{2} \cdot a n t_{-} s+\beta_{3} \cdot k p r_{\mathrm{it}}+\beta_{4} \cdot y_{2}+\cdots+\beta_{12} \cdot y_{10}+\epsilon_{\mathrm{it}}$

Die fünfte und letzte Spezifikation unterscheidet sich von der vierten nur dahingehend, dass die Standardfehler robust geschätzt werden. ${ }^{19}$

Diese letzte, von uns bevorzugte Spezifikation wurde schließlich zwei Sensitivitätschecks unterzogen. Zum einen wurde überprüft, ob evtl. auftretende Messfehler bzw. -ungenauigkeiten (der erhobenen Daten) unsere Schätzergebnisse verzerren. Werden Variablen fehlerhaft gemessen, so wird die Ungenauigkeit der Schätzung bei der Fixed-Effects-Regression im Vergleich zu einer KQ-Schätzung deutlich verstärkt. Dies wurde mittels eines Vergleichs von „Fixed-Effects“- und „First-Differenced“-Ergebnissen überprüft. Von dem Vergleich ausgehend zeigen sich die zentralen Variablen sehr stabil, weshalb von messfehlerfreien, unverzerrten Ergebnissen ausgegangen werden kann. Zum anderen wurde dem Fakt Rechnung getragen, dass Autokorrelation im Fehlerterm ein häufig auftretendes Phänomen bei Panelanalysen ist, welches die Schätzergebnisse unter Umständen stark beeinflussen kann. Eine separat durchgeführte Panelregression, welche

16 Weil die Abhängige Variable ein pro-Kopf Maß ist, wird hier nicht der Gesamtpräventionsbetrag, sondern die Prävention je Versicherten verwendet.

17 Wären die Präventionsausgaben Teil der Verwaltungs- und Verfahrenskosten, müsste man in einer weiteren Spezifikation die abhängige Variable dahingehend modifizieren, dass die Präventionskosten aus den Verwaltungs- und Verfahrenskosten heraus gerechnet werden. Dies ist allerdings nicht notwendig, da aus den Geschäftsberichten der Unfallkassen eindeutig hervorgeht, dass beide Kostenarten separat in verschiedenen Kontenklassen erfasst und nicht miteinander verrechnet werden.

18 Um besondere Einflüsse einzelner Perioden wie z. B. die Folgejahre der Fusionswelle 1998 auf die Schätzergebnisse ausschließen zu können, wurden testweise einzelne bzw. mehrere (bis zu drei) Jahre aus der Schätzung entnommen. Die Größenordnung der Parameter (siehe Kapitel 5) blieb hierdurch unberührt.

19 Die sich daraus ergebenden Standardfehler sind übereinstimmend mit über die Kassen gruppierten Standardfehlern. 
bewusst für einen autoregressiven Fehlerterm erster Ordnung kontrolliert, zeigt jedoch, dass die wesentlichen Variablen unseres Modells fehlerfrei geschätzt wurden. Daher werden im weiteren Verlauf ausschließlich die Ergebnisse der ursprünglich vorgesehenen Spezifikationen dargestellt und diskutiert.

\section{Ergebnisse}

In Tabelle 1 sind die Ergebnisse der Fixed-Effects-Regression abgebildet. Die erste Spezifikation erklärt die Verwaltungs- und Verfahrenskosten ausschließlich durch die Anzahl der Versicherten. Der Koeffizient für die relevante Größe (Versicherte gesamt in Tausend) liegt bei ca. - 0,3 Cent und ist zum $1 \%$-Niveau statistisch signifikant.

Modell 2 berücksichtigt auch den prozentualen Anteil der Schüler an der Anzahl Versicherte gesamt. Erwartungsgemäß trägt der Koeffizient ein negatives Vorzeichen. Ein prozentualer Anstieg des Schüleranteils an den Versicherten hat damit einen Rückgang der Verwaltungs- und Verfahrenskosten zur Folge.

Führen wir in der dritten Spezifikation die Präventionsintensität (gemessen in Präventionsausgaben je Versicherten) ein, so schrumpfen die anderen beiden Koeffizienten auf etwa die Hälfte. Die Präventionsausgaben zeigen sich als höchst signifikant unterschiedlich von null, allerdings ist der Koeffizienten mit einem Wert von ca. 1,3 sehr groß und damit nicht glaubwürdig. Dies würde bedeuten, dass sich die Verwaltungs- und Verfahrenskosten pro Versicherten einer Unfallkasse bei Präventionsmehrausgaben von einem Euro im Schnitt um 1,30 Euro erhöhen.

Werden weitere Kontrollvariablen in Form von Jahresdummies eingeführt (Modell 4), bleibt die Variable Versicherte Gesamt, sowohl was die Größe des Koeffizienten (ca. -0,3 Cent) als auch dessen Signifikanzniveau angeht, stabil im Vergleich zur dritten Spezifikation. Der Koeffizient der Präventionsintensität nähert sich einem realistischen Niveau an und bleibt nach wie vor zum 1\%-Niveau signifikant. Nun steigen die Verwaltungs- und Verfahrenskosten pro Versicherten einer Unfallkasse im Schnitt um fast 80 Cent an, wenn 1 Euro mehr für Prävention ausgegeben wird. Dieses Ergebnis erscheint im Vergleich zur vorherigen Spezifikation plausibler. Der Anteil der Schüler in Prozent an allen Versicherten signalisiert, dass die Kosten je Versicherten um 4 Cent sinken, wenn der Anteil der Schüler an allen Versicherten um 1\% größer ist. Dies belegt, dass Schüler weniger Kosten verursachen als allgemein Versicherte (vgl. Kapitel 2).

Die fünfte Spezifikation unterscheidet sich lediglich dahingehend von letzterer, dass die Standardfehler nun robust geschätzt werden. Hier wird ersichtlich, dass sowohl der Effekt der Skalenerträge als auch der Effekt der Präventionsintensität nach wie vor signifikant bleiben, wenngleich jeweils zu einem schwächeren statistischen Signifikanzniveau (5\% bzw. 10\%).

Über alle durchgeführten Spezifikationen hinweg können somit die erwarteten Skalenerträge identifiziert werden. Dieses Ergebnis ist über die unterschiedlichen Spezifikationen stabil und auch im umfangreichsten und robust geschätzten Modell nachweisbar. Die Ergebnisse weisen deutlich auf Einsparpotentiale durch Zusammenschlüsse hin.

Das tatsächliche Ausmaß des Koeffizienten für Skalenerträge lässt sich am besten durch eine beispielhafte Kalkulation veranschaulichen. Der Mittelwert der Variable Versicherte gesamt (in Tausend) liegt bei 959 mit einer Standardabweichung von 603 (vgl. Tabelle 2). Eine Steigerung 
der Kassengröße - gemessen in Versicherten gesamt - um eine Standardabweichung würde also zu folgender Ersparnis führen:

$\mathrm{SD}_{\mathrm{vvjv}} * \beta_{1}=$ Ersparnis

$603 * 0.00329 \approx 1,98 €$

Damit ließen sich bei der Vergrößerung einer Versicherung um eine Standardabweichung $1,98 €$ pro Versicherten einsparen. Bei einem Mittelwert von 6,54 $€$ Kosten pro Versicherten über die verschiedenen Unfallkassen wäre das eine potentielle Ersparnis von ca. 30\%.

Panel-Regression: Abhängige Variable: V\&V Kosten je Versicherten

\begin{tabular}{|c|c|c|c|c|c|}
\hline \multirow{2}{*}{ VARIABLEN } & (1) & (2) & (3) & (4) & (5) \\
\hline & $\mathrm{FE}$ & $\mathrm{FE}$ & $\mathrm{FE}$ & FE & FE robust \\
\hline \multirow[t]{2}{*}{ Versicherte gesamt in Tausend } & $-0.00295^{* * *}$ & $-0.00508 * * *$ & $-0.00263^{* * *}$ & $-0.00329 * * *$ & $-0.00329^{*}$ \\
\hline & $(0.001000)$ & $(0.000960)$ & $(0.000625)$ & $(0.000629)$ & $(0.00183)$ \\
\hline \multirow{2}{*}{$\begin{array}{l}\text { Anteil der Schüler in Prozent an allen Ver- } \\
\text { sicherten }\end{array}$} & & $-0.175^{* * *}$ & -0.0225 & $-0.0465 * *$ & $-0.0465^{*}$ \\
\hline & & $(0.0272)$ & $(0.0196)$ & $(0.0198)$ & $(0.0242)$ \\
\hline \multirow[t]{2}{*}{ Präventionsausgaben je Versicherten } & & & $1.323 * * *$ & $0.801 * * *$ & $0.801 * *$ \\
\hline & & & $(0.0812)$ & $(0.164)$ & $(0.304)$ \\
\hline Jahresdummies & Nein & Nein & Nein & $\mathrm{Ja}$ & $\mathrm{Ja}$ \\
\hline \multirow[t]{2}{*}{ Konstante } & $9.367 * * *$ & $24.81 * * *$ & $7.517 * * *$ & $11.00^{* * *}$ & $11.00^{* * *}$ \\
\hline & $(0.960)$ & $(2.550)$ & (1.930) & (1.966) & $(3.603)$ \\
\hline Beobachtungen & 198 & 198 & 198 & 198 & 198 \\
\hline $\mathrm{R}^{2}$ & 0.047 & 0.229 & 0.694 & 0.739 & 0.739 \\
\hline
\end{tabular}

Tabelle 1: Fixed Effects Regressionsoutput - Übersicht Standardfehler in Klammern

Standardfehler in Klammern

$* * * \mathrm{p}<0.01, * * \mathrm{p}<0.05, * \mathrm{p}<0.1$

\section{Fazit und Ausblick}

Aus den Ergebnissen der durchgeführten Regressionsanalyse über den Zeitraum von 10 Jahren und 20 Kassen können zwei wesentliche Schlüsse gezogen werden.

Zunächst konnte die Existenz von Skaleneffekten bei den öffentlichen Unfallkassen in jeder durchgeführten Spezifikation belegt werden. In der bevorzugten (dritten bzw. vierten) Spezifikation beträgt der Koeffizient für die Anzahl der Versicherten in Tausend -0.33 Cent und ist statistisch signifikant. Die Ergebnisse lassen sich auf politischer Ebene in doppelter Hinsicht bewerten. Einerseits kann die Existenz von Skalenerträgen als positives Indiz zur Einschätzung aller, bereits durchgeführter Zusammenschlüsse dienen. Andererseits zeigen die Ergebnisse, dass die von Seiten der Politik eingeschlagene Richtung (weitere Konzentration) grundsätzlich sinnvoll 
ist. Zwar kann auf Basis von Daten der Jahre 1998-2007 keine Evaluation der Umsetzung des UVMG durchgeführt werden, die Intention der Gesetzgeber ist allerdings auf Basis der vorliegenden empirischen Ergebnisse positiv zu bewerten.

Im Vergleich mit den Ergebnissen der Querschnittsanalyse von Kaul und Haag (2008) ergibt sich somit ein gegenläufiges Bild. Bereinigt um etwaige Störfaktoren wie die mögliche Heterogenität zwischen den Kassen und über die Zeit und darüber hinaus ausgeweitet auf eine Paneldimension, liefert die Fixed-Effects-Schätzung ein durchweg plausibles Ergebnis: Größere Kassen arbeiten letztlich effizienter als kleine. Des Weiteren gibt es neben den gemeldeten und meldepflichtigen Unfällen noch weitere Kostentreiber, die einen signifikanten Einfluss auf die Verwaltungs- und Verfahrenskosten der öffentlichen Unfallkassen haben. So scheint insbesondere der Effekt von Präventionsausgaben je Versicherten auf wissenschaftlicher Ebene interessant. Der Quotient von 0.689 besagt, dass die Verwaltungs- und Verfahrenskosten mit jedem zusätzlich für Prävention ausgegebenem Euro um 69 Cent steigen. Dieses Ergebnis deckt sich mit dem zweiten Hauptergebnis der Untersuchung von Kaul und Haag (2008).

Der negative Effekt von Präventionsausgaben auf die Effizienz der Verwaltung kommt an dieser Stelle damit nicht überraschend, ist aber vor dem Hintergrund zweier weiterer Fragen von Relevanz. Sind auch hier Skaleneffekte zu beobachten und werden die Kosten der Prävention durch ihren Nutzen überkompensiert? Die Beantwortung dieser Fragen würde den Umfang der vorliegenden Analyse sprengen, bietet aber Potential für weitergehende Forschung.

\section{Abstract}

Ashok Kaul, Gregor Pfeifer, Manuel Schieler and Stefan Witte; Economies of Scale in Public Statutory Accident Insurance Funds in Germany

Administration and Management Costs; Economies of Scale; German Statutory Accident Insurance; Panel Data Analysis; Savings Potential; Statutory Accident Insurance Modernization Act

As of January 1, 2009, the German Public Statutory Accident Insurance was fundamentally reformed within the framework of the Statutory Accident Insurance Modernization Act. A central goal of the act was the reduction of administration and management costs per insured person, which basically implies realizing economies of scale. However, there was no empirical evidence for economies of scale in this sector at that time. Using data from 1998-2007, the present study provides a first answer to the question whether bigger accident insurers have lower administrations and management costs per insured person than smaller ones. Applying panel data methods, we can show that those costs are adversely affected by the size of the cost bearer. The results are robust to alternative specifications. On average, the administration and management costs per insured person would be reduced by 33 Eurocent if the number of insured individuals increased by 100'000. At medium costs of $€ 6.54$ per insured individual and 960.000 insured per accident insurer on average there is a considerable savings potential. An increase in size of about 600.000 insured (one standard deviation) would lead to about $30 \%$ of cost reduction. 


\section{Literaturverzeichnis}

Albers, S./Klapper, D./Konradt, U./Walter, A./Wolf, j. (2009): Methodik der empirischen Forschung, 3. Auflage, Wiesbaden 2009.

Baltagi, B.H. (2008): Econometric Analysis of Panel Data. 4. Auflage, Chichester 2008.

Bund-Länder-Arbeitsgruppe (2006): Beschluss der Bund-Länder-Arbeitsgruppe „Eckpunkte zur Reform der gesetzlichen Unfallversicherung“. 2006.

Greene, W. (2008): Econometric Analysis. 6. Auflage, New Jersey 2008.

Kaul, Ashok und Lukas Haag (2008), Reformen auf der Suche nach Skaleneffekten: Die Struktur der Verwaltungsund Verfahrenskosten der öffentlichen Unfallkassen in Deutschland, in: Zeitschrift für öffentliche und gemeinwirtschaftliche Unternehmen (ZögU), 3/2008, 249ff.

Kennedy, P. (2009): A Guide to Econometrics. 6. Auflage, Malden 2009.

Roland Berger Strategy Consultants (2005): Auswirkungen möglicher Reformoptionen in der gesetzlichen Unfallversicherung. Gutachten im Auftrag des Landes NRW, Hamburg 2005.

Rürup, B./Steinmeyer, H.-D. (2006), Gutachten zur Neuorganisation der gesetzlichen Unfallversicherung. Online Version vom 30. März 2006, Münster und Darmstadt 2006.

Sopoaktuell (2006), Reform der gesetzlichen Unfallversicherung, Positionsbestimmung des ver.di-Bundesvorstandes, Nr. 40, 25. April 2006.

\section{Anhang}

\begin{tabular}{|l|l|c|c|c|c|}
\hline Variable & \multicolumn{1}{|c|}{ Label } & MW & StAbw & Min & Max \\
\hline kvv & $\begin{array}{l}\text { Verwaltungs-und Verfahrens- } \\
\text { kosten (in } € \text { ) je Versicherten }\end{array}$ & 6539.46 & 1531.79 & 2730.52 & 10918.26 \\
\hline VAUV & Anzahl Versicherte AUV & 225.83 & 167.28 & 42.51 & 805.94 \\
\hline VSUV & Anzahl Versicherte SUV & 730.24 & 449.92 & 147.45 & 1671.23 \\
\hline VGES & Anzahl Versicherte gesamt & 959.33 & 603.45 & 203.27 & 2477.17 \\
\hline KAUV & $\begin{array}{l}\text { Kosten (in } € \text { ) Verwaltungs- und } \\
\text { Verfahrenskosten AUV }\end{array}$ & 5901386.00 & 3662341.00 & 1271092.00 & 14700000.00 \\
\hline KSUV & $\begin{array}{l}\text { Kosten (in } € \text { ) Verwaltungs- und } \\
\text { Verfahrenskosten SUV }\end{array}$ & 102822.20 & 75821.05 & 8873.35 & 377445.80 \\
\hline KVV & $\begin{array}{l}\text { Kosten (in } € \text { ) Verwaltungs- und } \\
\text { Verfahrenskosten gesamt }\end{array}$ & 6004208.00 & 3728684.00 & 1288388.00 & 15000000.00 \\
\hline kpr & $\begin{array}{l}\text { Kosten (in } € \text { ) für Präventions- } \\
\text { ausgaben je Versicherten }\end{array}$ & 2468.60 & 742.08 & 373.93 & 4623.42 \\
\hline KPR & $\begin{array}{l}\text { Kosten (in } € \text { ) für Präventions- } \\
\text { ausgaben gesamt. }\end{array}$ & 2279796.00 & 1628575.00 & 435472.70 & 7180493.00 \\
\hline ant_s & $\begin{array}{l}\text { Anteil der Schüler an allen Ver- } \\
\text { sicherten }\end{array}$ & 76.44 & 4.92 & 67.08 & 87.61 \\
\hline
\end{tabular}

Tabelle 2: Zusammenfassende Statistiken 